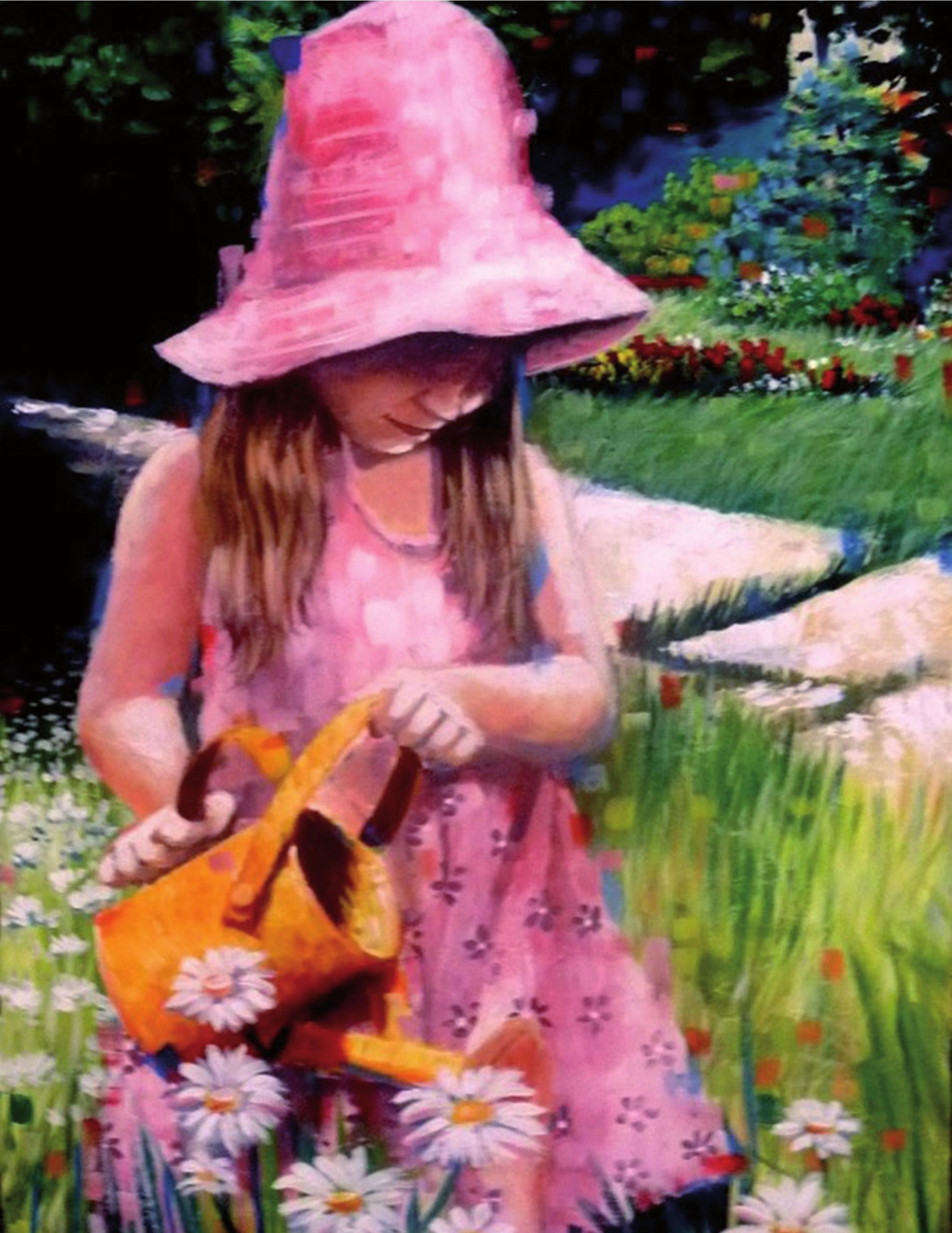




\title{
Mortalidad infantil y el acceso al recurso humano de salud. Tres años de revisión; 2009-2011
}

María Guadalupe Romero-A., Ingrid Carolina Garay-R. *

\begin{abstract}
RESUMEN. La disponibilidad de programas orientados a disminuir la mortalidad, sobre todo, por causas prevenibles define vínculos importantes, como la de garantizar la disponibilidad de proveedores. La mortalidad infantil ha ido disminuyendo con diferentes estrategias que los países cimentan con este objetivo. El presente trabajo es una investigación descriptiva retrospectiva que toma los datos del Boletín Epidemiológico de la Secretaría de Salud de Honduras en cuanto a los datos de mortalidad infantil en el período 2009 a 2011 y los datos que se encuentran en el Anuario Estadístico de la Secretaría de Salud de los años en mención para el recurso humano hospitalario y no hospitalario, específicamente médicos, profesionales de enfermería y auxiliares de enfermería. Los resultados señalan que el año con mayor cantidad de muertes infantiles fue el año 2011. Los departamentos con mayores tasas de mortalidad en los tres años, fueron Francisco Morazán, Cortés, Olancho. Las mayores tasas de cobertura del recurso humano para la población en general, se encuentran en los departamentos de Islas de la Bahía, Atlántida y Valle. Los que presentaron menor oportunidad de cobertura para este grupo son: Santa Bárbara y El Paraíso. Para el grupo específico de menores de un año, los departamentos que tienen mayor oferta son: Islas de la Bahía, Gracias a Dios, Francisco Morazán. Los que menos ofertan son: El Paraíso, Santa Bárbara, Olancho e Intibucá.
\end{abstract}

Palabras claves: mortalidad infantil, recurso humano, acceso.

ABSTRACT. The availability of programs designed to reduce mortality, especially from preventable defines important links, such as ensuring the availability of providers. Infant mortality has declined over different strategies that underpin countries for this purpose. The present work is a retrospective descriptive research data takes Epidemiological Bulletin of the Ministry of Health of Honduras regarding infant mortality data for years 2009-2011 and the data found in the Statistical Yearbook of the Ministry of Health of the years in question for human resources and hospital inpatient, specifically doctors, nurses and nursing assistants. The results indicate that the year with the highest number of infant deaths was 2011. The departments with the highest mortality rates in the three years were Francisco Morazán, Cortes, Olancho. The highest rates of coverage of human resources for the general population, are in the departments of the Bay Islands, Atlantis and Valle. The less chance we have coverage for this group are: Santa Barbara and El Paraíso. For the specific group under one year, the departments that have greater offer are: Bay Islands, Gracias a Dios, Francisco Morazán. The least offered are: El Paraíso, Santa Barbara, Olancho, Intibucá.

Keywords: infant mortality, human resources, access.

\section{Introducción}

Existen vínculos importantes entre la salud y el desarrollo de los países. Entre esos vínculos se encuentra, la disponibilidad de programas muy bien orientados para disminuir la mortalidad, sobre todo, por causas prevenibles y otro vínculo importante es garantizar la disponibilidad de proveedores y de infraestructura (Rodríguez Acosta, 2010).

A nivel de todos los países del mundo, la mortalidad ha ido disminuyendo independientemente de las dife- rencias económicas y las diferentes estrategias que los países cimentan en ese aspecto. Entre estas estrategias se permite mencionar la vacunación que se lleva a los hogares en forma directa, obstaculizando a partir de esta acción, las causas que en un momento determinado, se establecieron como únicas.

El logro se estableció con los datos en un período de tiempo determinado, sin embargo, posteriormente en los países en vías de desarrollo, se plasma con mayor precisión requeriendo revisar otro tipo de determinantes, que no fueran la prevención de ciertas enfermedades, las

Recibido: abril 2014/Aceptado y versión final agosto 2014

* Médico y Cirujano. Máster en Salud Pública. Máster en Administración de Servicios de Salud. PhD.

** Ingeniera en Ciencias Ambientales. Máster en Ciencias, Tecnología y Gestión del Agua .PhD. 
que también tenían presencia en la mortalidad y en este caso la mortalidad infantil.

La Cumbre Mundial a favor de la Infancia fijó para el año 2000 metas precisas y para realizarlas, requería evaluaciones que permitieran visualizar los avances en las mismas. La información fue necesaria para que cada país pudiera asignar recursos y obtener resultados con eficiencia (Ahmad, López, \& Inoue, 2000).

Con esto se logró que aumentaran los datos disponibles y se evidenció el aumento o la disminución de las tasas de mortalidad infantil en regiones y países.

El presente trabajo realiza una revisión documental de los datos de mortalidad infantil que aparecen en el Boletín Epidemiológico del año 2009 al año 2011 de la Secretaría de Salud y lo relaciona con los datos que el Sistema de Salud proporciona a través de los Anuarios Estadísticos de los años en observación, logrando relacionar la mortalidad notificada con la disponibilidad del recurso humano en salud.

\section{Aspectos conceptuales}

\subsection{Mortalidad infantil}

Es una variable demográfica que indica el número de niños menores de un año fallecidos a lo largo de un período de tiempo determinado. También se define como la razón de defunciones a la edad de 0 a 1 años, frente a los nacimientos del mismo período (Urdinola, 2011). Por lo general, la mortalidad infantil, se expresa como tasa y es aquella que da la proporción existente entre el número de niños menores de un año fallecidos en el curso de un año y el número de nacimientos vivos durante el mismo año, se expresa en tanto por mil y se puede agrupar por localidad, país y sexo. La Secretaría de Salud de Honduras, define la mortalidad infantil, como la muerte de un niño (a) en la edad comprendida entre los 0 y 11 meses 29 días (Honduras, 2008).

La tasa de mortalidad infantil es uno de los indicadores demográficos que mejor refleja el contexto socioeconómico de un país. Amén de ser un indicador efectivo en describir las condiciones de mortalidad, la
TMI es muy eficiente en capturar diferentes problemas de bienestar social y de desarrollo socioeconómico de cualquier población que se asocia a las mejoras en capital físico (infraestructura y hospitales) y humano (como la educación de los padres) que debe hacer una sociedad por mejorar las condiciones de vida.

La Organización Mundial de la Salud (OMS) ha puesto énfasis en los determinantes sociales de la salud, de tal forma, que a partir del año 2005, crea la Comisión de Determinantes Sociales de la Salud (CDSS) con el fin de aportar conocimientos y sensibilizar sobre el tema. Los ejes de la desigualdad están relacionados con el nivel socioeconómico y pueden expresarse bajo el nivel de ingresos, nivel de instrucción, lugar de residencia (Dirección de Planificación, 2009).

Las desigualdades en salud, son diferencias en salud producidas socialmente que se distribuyen de manera sistemática entre la población y que son injustas (OMS C. d., 2007).

En ese sentido, los sistemas de salud, tienen la obligación de ser asequibles, equitativos, accesibles, sostenibles y de buena calidad, sin embargo, bajo la visión de la (OMS), ciertos objetivos deseados como la accesibilidad, son un medio para alcanzar un fin, son metas instrumentales. Cuanto más accesible sea un sistema, más debería de utilizarlo la gente, para mejorar su salud (Rodríguez, 2010).

De acuerdo con los principios de equidad sanitaria, todos los ciudadanos deberían tener las mismas oportunidades de gozar de un buen estado de salud, sin embargo, en muchos países existen grandes desigualdades. El no lograr acceder a prestadores de atención sanitaria capacitados, es una de las primeras causas de inequidad sanitaria y adquiere importantes dimensiones en las zonas rurales (OPS, 1998).

La literatura reporta una relación positiva entre la supervivencia infantil y el acceso a los servicios de salud; pocos estudios han incluido dentro de sus determinantes, alguna medida de utilización de esos servicios. El estudio de Forste en 1994 estimó para Bolivia el impacto de la variable atención recibida por un profesional de salud 
(médico, enfermera u obstetriz) sobre el riesgo de morir, encontrando una relación positiva aun cuando no significativa estadísticamente, mientras que otros factores como atención prenatal, si lo son. Un estudio efectuado por el INEI para el caso peruano reporta que el parto atendido por un profesional de la salud u otro técnico reduce significativamente el riesgo de morir, siendo el resultado estadísticamente significativo (Dammert, 2001).

Estudios como el de Panis y Lillard en 1995, estiman mediante una ecuación de riesgos proporcionales una ecuación de mortalidad infantil junto con dos ecuaciones de demanda por atención médica, es decir, incorporan en la ecuación de mortalidad infantil la probabilidad de atención médica y lugar de atención del parto (et al, 2001).

\subsection{Recurso humano en salud}

Existe una escasez crónica de personal sanitario bien preparado. Tiene carácter mundial pero es de especial gravedad en los países que más los necesitan. El factor humano es un elemento indispensable para reforzar los sistemas de salud. Abundan las pruebas de que el número y la calidad de los trabajadores sanitarios están efectivamente relacionados con la supervivencia de los lactantes y de los niños. Se ha constatado que la combinación de capacidades de los equipos sanitarios varía enormemente de unos países a otros. El número de enfermeras por cada médico en el Canadá y en los Estados Unidos de América es de aproximadamente 4 enfermeras por cada médico frente a menos de una en Chile, Perú, El Salvador y México (OMS, 2006). En relación a la distribución del recurso humano por área geográfica, son muchos los factores que influyen en la densidad del personal sanitario. Las zonas donde existen hospitales docentes y cuya población cuenta con medios suficientes para pagar servicios de salud, atraen a más trabajadores de salud que aquellas que carecen de este tipo de instituciones y de los medios de financiamiento, razón por la cual, las áreas urbanas se ven favorecidas. (OMS, 2006).

\section{Metodología}

Se trata de un diseño de investigación descriptivo y retrospectivo.

Se revisaron los datos proporcionados por el Boletín Epidemiológico de la Dirección de Vigilancia de la Salud del año 2009 al 2011 con respecto a mortalidad infantil por departamentos y los datos proporcionados en el Anuario Estadístico de la Secretaría de Salud, de los años 2009 al 2011, con respecto al recurso humano.

Las variables analizadas fueron: mortalidad infantil por región departamental, recurso humano médico, recurso humano de profesionales de enfermería, recurso humano auxiliar de enfermería, recurso humano hospitalario y no hospitalario. Se agrupó en una sola categoría denominada enfermería a las profesionales de la enfermería y a las auxiliares de enfermería. El recurso humano no hospitalario que incorpora a los datos, contempla las categorías médico y enfermería.

\section{Resultados de la investigación}

Honduras, ha evidenciado un descenso importante en la tasa de mortalidad infantil, pero este descenso es más lento que en otros países de la Región Centroamerica; considerándolo como uno de los logros más importantes en la Salud Pública. Los determinantes en el país, están relacionados con el área geográfica, siendo más vulnerable el área rural; el nivel educativo alcanzado por la madre, de tal forma que los niños cuyas madres no tienen educación, tienen dos veces más riesgo de morir en el primer año de vida, que la de aquellos niños cuyas madres tienen educación superior. El índice de riqueza es también determinante en la mortalidad y los datos señalan que la mortalidad en los hogares en el quintil inferior es el doble comparado con el estrato superior; de igual forma, existe una fuerte relación entre fecundidad y mortalidad infantil; si disminuye la fecundidad, disminuyen mucho los factores de alto riesgo para la mortalidad infantil: embarazos en edades avanzadas, intervalos con el parto anterior cortos (UNFPA, 2009). 
Los resultados permiten ver la notificación de casos de mortalidad infantil en los tres años sujetos a observación: 2009 a 2011, los promedios de muerte por año por departamento, los rangos de los datos por cada año por región departamental de salud, la velocidad de muerte por semana epidemiológica, así como las tasas de mortalidad infantil por departamento por año, las tasas de recurso humano de salud por población total por año y las tasas de recurso humano por grupo específico de análisis que corresponde a los menores de un año por región departamental.

El año 2011, reporta la mayor cantidad de notificaciones de mortalidad infantil en el período de tres años sujetos a observación. En ese año, el primer lugar lo ocupa el departamento de Francisco Morazán, continuando el departamento de Cortés que ocupa el segundo lugar y Olancho en el tercer lugar y Choluteca enel cuarto lugar. La velocidad de muerte es de 54 muertes por semana epidemiológica. El rango de los datos para este año es de
8 (valor mínimo) a 872 (valor máximo). En el año 2010, el primer lugar lo ocupa el departamento de Francisco Morazán, el segundo lugar el departamento de Cortés, el tercer lugar el departamento de Copán y el cuarto lugar, Olancho. La velocidad de muerte es de 52 muertes por semana epidemiológica. El rango de los datos es de 11 (valor mínimo) a 764 (valor máximo). En el año 2009, el primer lugar lo ocupa el departamento de Francisco Morazán, el segundo lugar, el departamento de Cortés, el tercer lugar, el departamento de Copán y el cuarto lugar, Choluteca. La velocidad de muerte es de 43 muertes por semana epidemiológica. El rango de los datos se encuentra de 8 (valor mínimo) a 684 (valor máximo) (Cuadro 1 y Gráfico 1).

Para el año 2009 a nivel de la población menor de 1 año los departamentos con mayor oportunidad de ofertar recurso humano médico son: Francisco Morazán, Gracias a Dios, Islas de la Bahía, Ocotepeque y Valle. Desde el punto de vista de enfermería, los departamentos que ofer-

\section{Cuadro 1. Casos notificados de mortalidad infantil por departamento, años 2009-2011}

\begin{tabular}{|c|c|c|c|c|c|c|}
\hline \multirow[b]{2}{*}{ DEPARTAMENTO } & \multicolumn{2}{|c|}{2009} & \multicolumn{2}{|c|}{2010} & \multicolumn{2}{|l|}{2011} \\
\hline & CASOS NOTIFICADOS & PORCENTAJES & CASOS NOTIFICADOS & PORCENTAJES & CASOS NOTIFICADOS & PORCENTAJES \\
\hline Atlántida & 83 & 3.75 & 77 & 2.84 & 65 & 2.29 \\
\hline Colón & 48 & 2.16 & 88 & 3.24 & 86 & 3.04 \\
\hline Comayagua & 54 & 2.44 & 83 & 3.05 & 94 & 3.32 \\
\hline Copán & 163 & 7.36 & 174 & 6.42 & 161 & 5.69 \\
\hline Cortés & 483 & 21.83 & 641 & 23.67 & 647 & 22.8 \\
\hline Choluteca & 116 & 5.24 & 120 & 4.76 & 125 & 4.42 \\
\hline El Paraíso & 51 & 2.30 & 63 & 2.32 & 71 & 2.5 \\
\hline Francisco Morazán & 684 & 30.92 & 764 & 28.21 & 872 & 30.83 \\
\hline Gracias A Dios & 20 & 0.90 & 32 & 1.18 & 10 & 0.35 \\
\hline Intibucá & 85 & 3.84 & 84 & 3.10 & 98 & 3.46 \\
\hline Islas de la Bahía & 8 & 0.36 & 18 & 0.66 & 8 & 0.28 \\
\hline La Paz & 49 & 2.21 & 57 & 2.10 & 65 & 2.29 \\
\hline Lempira & 108 & 4.88 & 106 & 3.91 & 108 & 3.81 \\
\hline Ocotepeque & 37 & 1.67 & 57 & 2.10 & 44 & 1.55 \\
\hline Olancho & 109 & 4.92 & 150 & 5.53 & 181 & 6.4 \\
\hline Santa Bárbara & 35 & 1.58 & 93 & 3.43 & 78 & 2.75 \\
\hline Valle & 12 & 0.54 & 11 & 0.40 & 29 & 1.02 \\
\hline Yoro & 67 & 3.02 & 94 & 3.47 & 86 & 3.04 \\
\hline Total & 2,212 & 100.0 & 2,708 & & 2,828 & \\
\hline
\end{tabular}

Fuente: Secretaría de Salud, Boletín Epidemiológico. República de Honduras. 2009-2011 
Gráfico 1. Tasas de mortalidad infantil por departamento. Tres años: 2009-2011. (por mil habitantes)

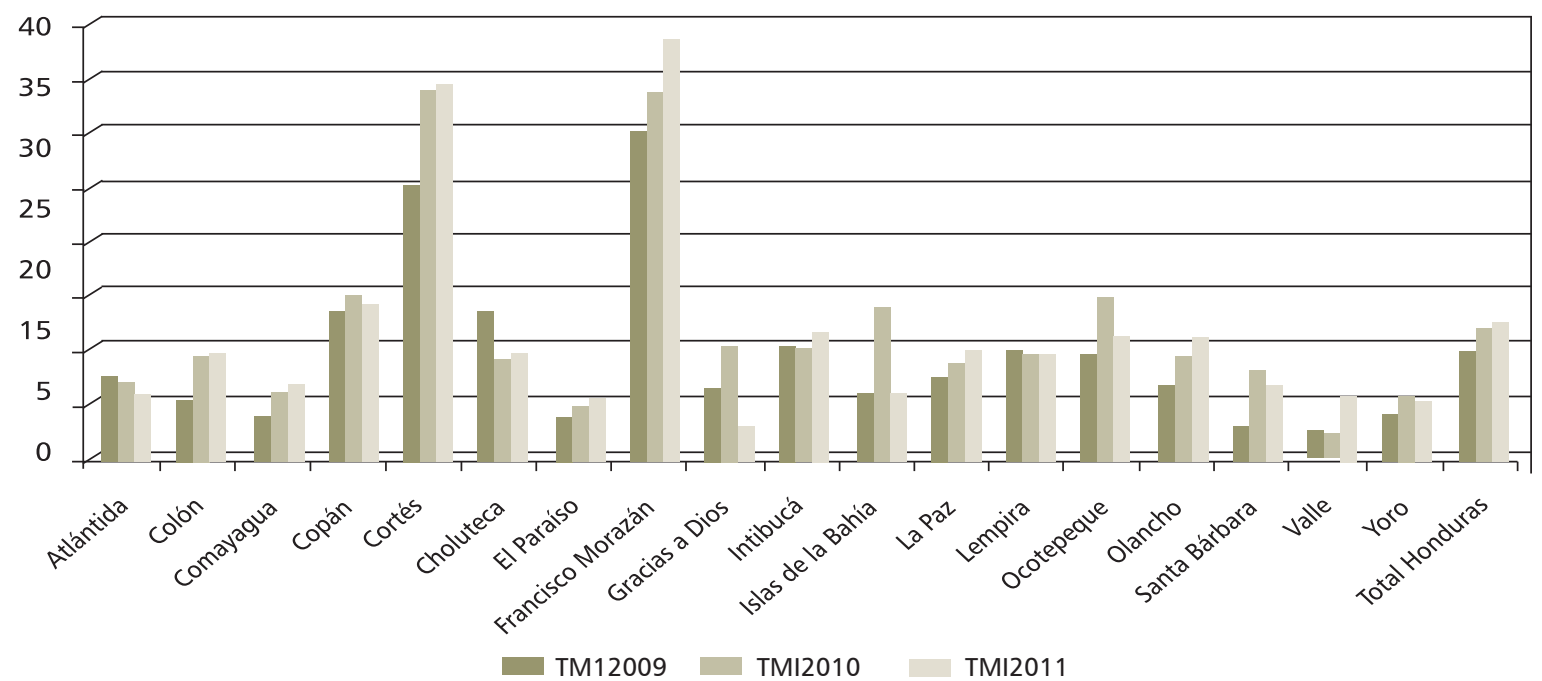

Fuente: Boletín Epidemiológico. República de Honduras. 2009.

tan más recurso son: Islas de la Bahía, Francisco Morazán, Ocotepeque y Valle. En ese año, el recurso de enfermería supera el de los médicos en una relación 2 a 4 veces más por cada 1,000 habitantes. Los que menos oferta brindan a nivel de médicos son: El Paraíso, Intibucá, Lempira, Olancho y Santa Bárbara. Estos tres departamentos presentan la misma tasa. A nivel de enfermeras, los departamentos con menos oportunidad son: Cortés, Santa Bárbara, El Paraíso, Olancho. En relación a la población total, la oferta del sistema de salud del recurso humano médico, se encuentra en los departamentos de Francisco Morazán, Choluteca e Islas de la Bahía. El recurso de enfermería, supera al médico a razón de 2 a 3 más enfermeras que médicos. Los que menos oportunidad tienen son: El Paraíso, Santa Bárbara, Olancho e Intibucá, pero el que refleja problemas en mortalidad infantil, es Francisco Morazán (Cuadro 2).

La tasa de recurso humano en el año 2010, para la población total en los departamentos que ofrecen mayor oportunidad de atención por parte de ellos son: Gracias a Dios con 1 médico por 1,000 habitantes; Islas de la Bahía, con aproximadamente 1 médico por 1,000 habitantes; Copán y Valle, con menos de 1 médico por 1,000 habi-
Cuadro 2. Tasa de recurso humano, año 2009

\begin{tabular}{|c|c|c|c|c|}
\hline \multicolumn{3}{|c|}{ POR MIL HABITANTES DE POBLACIÓN TOTAL } & \multicolumn{2}{|c|}{$\begin{array}{l}\text { POR MIL HABITANTES DE } \\
\text { POBLACIÓN MENOR DE } \\
1 \text { AÑO }\end{array}$} \\
\hline Departamento & Médico & Enfermera & Médico & Enfermera \\
\hline Atlántida & 0.33 & 0.8 & 14 & 35 \\
\hline Colón & 0.21 & 1 & 9 & 40 \\
\hline Comayagua & 0.22 & 0.8 & 9 & 33 \\
\hline Copán & 0.4 & 1 & 15 & 48 \\
\hline Cortés & 0.4 & 1 & 9 & 23 \\
\hline Choluteca & 0.6 & 2 & 13 & 47 \\
\hline El Paraíso & 0.1 & 0.6 & 6 & 26 \\
\hline Franco Morazán & 2.7 & 7 & 27 & 69 \\
\hline Gracias a Dios & 0.8 & 2 & 27 & 56 \\
\hline Intibucá & 0.2 & 0.8 & 8 & 30 \\
\hline Islas de la Bahía & 0.6 & 2 & 27 & 81 \\
\hline La Paz & 0.2 & 1 & 10 & 44 \\
\hline Lempira & 0.2 & 0.7 & 7 & 28 \\
\hline Ocotepeque & 0.4 & 1.4 & 16 & 61 \\
\hline Olancho & 0.2 & 0.7 & 7 & 28 \\
\hline Sta. Bárbara & 0.1 & 0.5 & 7 & 26 \\
\hline Valle & 0.4 & 1.3 & 16 & 60 \\
\hline Yoro & 0.2 & 0.8 & 10 & 37 \\
\hline
\end{tabular}

Fuente: Anuario Estadístico. Departamento de Planillas. Área de Sistemas de Información. Secretaria de Salud.2009 
tantes ocupando el tercer lugar. A nivel de enfermería, Islas de la Bahía ocupa el primer lugar, con 1.7 enfermeras por 1,000 habitantes; Valle con 1,2 y Atlántida, Colón, Copán, Choluteca, Francisco Morazán tienen la misma tasa de 1 por 1,000 habitantes. Entre los que menos ofertan, se encuentran: Yoro, Santa Bárbara, El Paraíso y Comayagua. En el año 2010 la oferta de médicos para el grupo de menores de un año, lo tiene en primer lugar el departamento de El Paraíso, Islas de la Bahía; el segundo lugar lo ocupa, Copán; el tercer lugar, Islas de la Bahía, Copán y Valle. El departamento que presenta la menor oferta es Francisco Morazán (Cuadro 3).

Los departamentos que ocupan los primeros lugares en la oferta de recurso humano médico para la población total, son: Gracias a Dios, Islas de la Bahía y Francisco Morazán. Los que ofertan menos, son: El Paraíso, Santa Bárbara, Lempira y Olancho, con menos de 1 médico por 1,000 habitantes. A nivel de enfermeras, la mayor

Cuadro 3. Tasa de recurso humano, año 2010

\begin{tabular}{|l|c|c|c|c|}
\hline \multicolumn{2}{|l|}{ POR MIL HABITANTES DE POBLACIÓN TOTAL } & \multicolumn{2}{|c|}{$\begin{array}{c}\text { POR MIL HABITANTES } \\
\text { DE POBLACIÓN MENOR } \\
\text { DE UN AÑO }\end{array}$} \\
\hline Departamento & Médico & Enfermera & Médico & Enfermera \\
\hline Atlantida & 0.3 & 1 & 15 & 42 \\
\hline Colón & 0.2 & 1 & 14 & 38 \\
\hline Comayagua & 0.2 & 0.8 & 12 & 33 \\
\hline Copán & 0.4 & 1 & 40 & 47 \\
\hline Cortés & 0.2 & 0.4 & 3 & 19 \\
\hline Choluteca & 0.3 & 1 & 6 & 46 \\
\hline El Paraíso & 0.1 & 0.6 & 88 & 47 \\
\hline Franco Morazán & 0.6 & 1 & 2 & 52 \\
\hline Gracias a Dios & 0.8 & 1 & 20 & 54 \\
\hline Intibucá & 0.2 & 0.8 & 5 & 30 \\
\hline Islas de la Bahía & 0.6 & 1.7 & 42 & 74 \\
\hline La Paz & 0.2 & 1 & 13 & 41 \\
\hline Lempira & 0.2 & 0.7 & 6 & 27 \\
\hline Ocotepeque & 0.3 & 1 & 30 & 59 \\
\hline Olancho & 0.2 & 0.7 & 4 & 28 \\
\hline Sta. Bárbara & 0.1 & 0.5 & 7 & 24 \\
\hline Valle & 0.4 & 1.2 & 32 & 56 \\
\hline Yoro & 0.2 & 0.5 & 11 & 23 \\
\hline
\end{tabular}

Fuente: Anuario Estadístico. Departamento de Planillas. Área de Sistemas de Información. Secretaria de Salud.2010 oferta la brindan los departamentos de: Islas de la Bahía, Atlántida, Colón, Copán, Choluteca. En este grupo de trabajadores de la salud los departamentos que menos ofertan son: Cortés, Santa Bárbara, Lempira y El Paraíso con menos de 1 por 1,000 habitantes (Cuadro 3).

En el año 2011, la oferta de recurso humano a nivel de médicos para el grupo poblacional de menores de un año, se privilegió para los departamentos de Francisco Morazán, Gracias a Dios e Islas de la Bahía. Los que menos oferta tienen son: El Paraíso, Santa Bárbara, Lempira, Intibucá. En cuanto al recurso humano de enfermería, el departamento que mayor oferta brinda es: Islas de la Bahía, Francisco Morazán, Ocotepeque, Valle. Los que menos oferta presentan son: Cortés, Santa Bárbara, El Paraíso, Olancho (Cuadro 4).

El análisis de los determinantes de la mortalidad infantil es relevante, no solo porque la salud tiene valor en sí misma, sino porque las condiciones de salud en la infancia

\section{Cuadro 4. Tasa de recurso humano, año 2011}

\begin{tabular}{|c|c|c|c|c|}
\hline \multicolumn{3}{|c|}{ POR MIL HABITANTES DE POBLACIÓN TOTAL } & \multicolumn{2}{|c|}{$\begin{array}{l}\text { POR MIL HABITANTES } \\
\text { DE POBLACIÓN MENOR } \\
\text { DE UN AÑO }\end{array}$} \\
\hline Departamento & Médico & Enfermera & Médico & Enfermera \\
\hline Atlantida & 0.3 & 1 & 15 & 43 \\
\hline Colón & 0.2 & 1 & 9 & 38 \\
\hline Comayagua & 0.2 & 0.7 & 9 & 33 \\
\hline Copán & 0.3 & 1 & 15 & 47 \\
\hline Cortés & 0.2 & 0.5 & 9 & 22 \\
\hline Choluteca & 0.3 & 1 & 13 & 46 \\
\hline El Paraíso & 0.1 & 0.6 & 6 & 25 \\
\hline Franco Morazán & 0.6 & 1 & 28 & 66 \\
\hline Gracias a Dios & 0.7 & 1 & 28 & 55 \\
\hline Intibucá & 0.2 & 0.8 & 8 & 30 \\
\hline Islas de la Bahía & 0.6 & 2 & 28 & 75 \\
\hline La Paz & 0.2 & 1 & 10 & 41 \\
\hline Lempira & 0.2 & 0.7 & 7 & 28 \\
\hline Ocotepeque & 0.3 & 1 & 15 & 59 \\
\hline Olancho & 0.2 & 0.7 & 7 & 28 \\
\hline Sta. Bárbara & 0.1 & 0.5 & 7 & 24 \\
\hline Valle & 0.3 & 1 & 16 & 57 \\
\hline Yoro & 0.2 & 0.7 & 11 & 33 \\
\hline
\end{tabular}

Fuente: Anuario Estadístico. Departamento de Planillas. Área de Sistemas de Información. Secretaria de Salud. Año 2011 
condicionan la salud en la adultez. No existe un consenso en relación a los factores que explican la mortalidad en general y en la mortalidad infantil, en particular. Sin embargo, se ha destacado la existencia de intervenciones públicas v.g. estrategias que han contribuido a disminuir la mortalidad infantil entre las que se puede mencionar: desarrollo y aplicación de las políticas públicas apropiadas y sostenibles y de mecanismos de protección social; fortalecer los sistemas de salud para asegurar el acceso en forma efectiva, incluyendo atención calificada del recién nacido organizando los servicios como respuesta a las demandas o cómo búsqueda de satisfacción de las necesidades de salud. Esto requiere que se realice un proceso de gestión que exige la observación cuidadosa de las muchas características peculiares del sector salud (Dussault \& Souza, 2000).

Filmer y Pritchett en el año de 1997, realizaron investigaciones y entre los resultados que encontraron destacan que deben tomarse en cuenta la eficiencia del sector público y el impacto neto de la provisión pública.

Abbas, en el año de 2009, encuentra que la disponibilidad y el acceso a los servicios de salud, son relevantes para determinar el estado de salud de la población, sobre todo en lo que se refiere a la tasa de mortalidad infantil (Tomado de Perazzo, 2011).

La mortalidad infantil, como problema de salud pública ha recibido un tratamiento central desde hace décadas. Se han realizado acciones y se han comprometido metas por parte de todos los países y de Honduras en particular, pero se debe realizar la búsqueda de los diferentes determinantes que se relacionan y que permitan fundamentar líneas de acción para alcanzar los propósitos establecidos tanto a nivel local, como a nivel nacional (OPS, 1998).

Honduras, en el año 2000, suscribe el compromiso de disminuir la mortalidad de los menores de 5 años, siendo la mortalidad infantil uno de los indicadores relevantes en este compromiso. De acuerdo al informe ODM 4 de Honduras, se ha llevado a cabo una reducción en la mortalidad infantil, la reducción de la misma no ha sido tan acelerada como requiere el cumplimiento de la meta al año 2015. Entre los obstáculos que se han presentado para llevar a buen recaudo la meta comprometida, se encuentra, el área de residencia urbana o rural, la deficiente cobertura hospitalaria y la calidad de los servicios de atención sanitaria y hospitalaria (UNPFA, 2010).

Basado en esa perspectiva, la atención sanitaria es una industria de servicios basada fundamentalmente en el capital humano. Los proveedores de atención en salud representan los valores esenciales del sistema porque curan y atienden a las personas. Son el vínculo humano entre el conocimiento y la acción sanitaria. La fuerza de trabajo es el elemento clave de todos los sistemas sanitarios para hacer progresar la salud. Existen pruebas contundentes de la relación entre el número y la calidad de los trabajadores sanitarios y la supervivencia de los lactantes y de los infantes.

Los trabajadores no son solo individuos sino que son componentes básicos de equipos sanitarios operativos en los que cada miembro aporta competencias distintas y desempeña funciones diferentes. La combinación de capacidades de los equipos sanitarios, varía entre los países, de tal forma que, la relación entre enfermeras y médicos varía de 8 a 1.5 en África, de 4 enfermeras por cada médico en Canadá y los Estados Unidos, frente a menos de una en Chile, Perú, El Salvador y México (OMS, 2006).

Los datos que se presentan en esta revisión establecen que la razón entre médico y enfermera en Honduras durante el período observado, es de 2 a 4 enfermeras por médico.

Uno de los aspectos relevantes, es la concentración del recurso humano en las zonas urbanas en detrimento de las rurales produciendo crisis de personal sanitario en las zonas que más requieren su presencia. Evidencia de esto, se observa en los departamentos de El Paraíso, Olancho e Intibucá. Los datos analizados proceden de las planillas de personal de acuerdo a nombramiento, pero no reflejan el movimiento del recurso humano una vez nombrado hacia áreas de alta concentración como ser las grandes urbes.

La OMS, ha identificado un umbral de densidad de personal sanitario por debajo del cual es muy improbable lograr una alta cobertura de intervenciones esenciales, 
como las necesarias para alcanzar los Objetivos de Desarrollo del Milenio (ODM), relacionados con la salud. La OMS, establece que en un mismo país, coexistan insuficiencias con un gran número de personal sanitario que por razones de falta de fondos públicos e interferencias políticas, generan escasez, generando una distribución inadecuada del recurso que no apoya expectativas de la población ni el cumplimiento de metas esenciales en grupos identificados de alta vulnerabilidad.

Los ODM, apuntan que los países más pobres están ligados a enfermedades que se centran en el grupo materno infantil, tal como ocurre en Honduras y las grandes dificultades para alcanzar las metas previstas, se relacionan con limitación del recurso humano o una inadecuada distribución del mismo.

Honduras, en ese sentido ha asumido compromisos desde el declaratorio de Alma Ata, en el año de 1978 en el cual se menciona el hecho de que la desigualdad existente en el estado de salud de la población, es política, social y económicamente inaceptable, incorporando por lo tanto, compromisos de acceso a los servicios de salud (OPS, 1978, 2009).

Uno de los aspectos más importantes de este acceso, es la presencia de recurso humano calificado. En la revisión de los tres años, conducida por el indicador de mortalidad infantil, se logra entrever que la distribución del recurso humano, sobre todo, el médico, decreció, aun en las zonas centrales y de alta producción económica, como departamento de Francisco Morazán y Cortés, que por el alto crecimiento de la población requerirían mayor contratación de personal para la cobertura adecuada de los servicios de salud y que en el contexto de las estrategias, la movilidad del personal se relacione para mejorar la cobertura, no obedeciendo a otras razones que no sean las que se encuentren acordes con los compromisos contraídos.

Los datos revelan que las tasas de cobertura tanto para la población general como para la población menor de un año, se mantuvo y no hubo crecimiento vegetativo del recurso humano, en aras del crecimiento de las poblaciones. También llama mucho la atención que con el mismo recurso, se logra ver el aumento de la tasa de mortalidad infantil en el último año. Este dato, debe llamar a la reflexión. Es sabido que hay varios aspectos que se relacionan con la mortalidad de los niños en su primer año de vida, pero lograr garantizar la presencia del recurso humano, debe ser una garantía así como la concienciación, el compromiso y el liderazgo político, asegurando de esta forma, la salud infantil.

Honduras, define en el marco Conceptual, Político y Estratégico de la Reforma del Sector Salud, que la exclusión es un fenómeno complejo y que la falta de acceso a los sistemas de salud destacan entre las causas que contribuye a condenar a amplios sectores de la población por lo que es importante establecer mecanismos impostergables que garanticen la protección social de la población a través de la accesibilidad a servicios de salud adecuados y eficientes (Honduras, 2009). No existe suficiente información y análisis sobre los problemas referidos a los recurso humanos en las reformas sectoriales de salud, teniendo como resultado que la política de recurso humano, no siempre surge de opciones explicitas y claras, sino de problemas fragmentados producto de la aplicación de los nuevos modelos de organización y financiamiento de la atención de la salud.

Se requiere construir una nueva perspectiva estratégica que privilegie las relaciones sociales e institucionales del sistema de salud. No se puede concebir ninguna transformación en ninguna de las dimensiones del sistema de servicios, sin considerar el papel fundamental que tiene el personal de salud. Este actor es indispensable para el logro de la calidad y la eficiencia de la atención, así como para una perspectiva política, asegurar los compromisos que asume el país en el contexto de los grupos vulnerables, como ser, los menores de un año (OPS, 2000). 


\section{Bibliografía}

- Ahmad, O., López, A., \& Inoue, M. (2000). Reevaluación de la disminución de la mortalidad infantil. Ginebra: Bulletin of the World Health Organization.

- Behm, H. (2011). Determinantes económicos y sociales de la mortalidad en América Latina. Buenos Aíres: CELADE.

- Dammert, A. C. (2001). Acceso a servicios de salud y Mortalidad Infantil. Lima: Consorcion de Investigación Económico y Social.

- Dirección de Planificación, E. (2009). Análisis de la mortalidad infantil según condicionantes sociales de la salud en la Provincia de Mendoza. Mendoza: Ministerio de Salud-Mendoza.

- Dussault, G., \& Souza, L. E. (2000). La Gestión Estratégica de los Recursos Humanos en Salud. Montreal: Facultad de Medicina.

- Honduras, S. d. (2009). Marco Conceptual Pólitico y Estratégico de la Reforma del Sector Salud 2009. Tegucigalpa.

- Honduras., S. d. (2008). Guía para la vigilancia de la mortalidad en el menor de 5 años en Honduras. Tegucigalpa.

- OMS. (2006). Colaboremos por la salud. Informe sobre la salud del mundo. Ginebra.

- OMS, C. d. (2007). Determinantes Estructurales de las Desigualdades de Salud. Ginebra.
- OPS. (1998). Mortalidad infatil un indicador para la gestión local. Washigton.

- OPS. (2000). Observatorio de los Recursos Humanos de Salud. Quito.

- OPS. (2009). Declaración de Alma Ata. Conferencia Internacional de Atención Primaria. Septiembre de 1978. Ginebra.

- Perazzo, I. (2011). Determinantes macroeconomicos de la mortalidad infantil en Uruguay. Insituto de Economía.

- PNUD. (1990). Desarrollo Humano. Informe 1990.

- PNUD. (2009). Desarrollo Humano en Honduras: limitado por la inequidad territorial. Tegucigalpa.

- Rodríguez Acosta, S. (2010). Barreras y Determinantes del Acceso a los Servicios de Salud en Colombia. Barcelona.

- Rodriguez, S. (2010). Barreras y Determinantes del Acceso a los Servicios de salud en Colombia. Barcelona.

- Salud, D. d. (2009). Boletín Epidemoológico de la República de Honduras. Tegucigalpa.

- UNFPA. (2009). Población y Salud. Análisis de Situación de Población en Honduras. Cuaderno 2.

- UNPFA. (2010). ODM4 Honduras. Tegucigalpa.

- Urdinola, P. (2011). Determinantes socieconómicos de la mortalidad infantil en Colombia, 1993. Bogotá: Revista Colombiana de Estadística. Vol 34.No.1,pp 39 a 72. 